\title{
Review Article \\ Robotics for Natural Orifice Transluminal Endoscopic Surgery: A Review
}

\author{
Xiaona Wang and Max Q.-H. Meng \\ Department of Electronic Engineering, The Chinese University of Hong Kong, Shatin, NT, Hong Kong \\ Correspondence should be addressed to Xiaona Wang, xnwang@ee.cuhk.edu.hk
}

Received 11 September 2012; Accepted 18 December 2012

Academic Editor: Yangmin Li

Copyright (c) $2012 \mathrm{X}$. Wang and M. Q.-H. Meng. This is an open access article distributed under the Creative Commons Attribution License, which permits unrestricted use, distribution, and reproduction in any medium, provided the original work is properly cited.

\begin{abstract}
Natural Orifice Transluminal Endoscopic Surgery (NOTES) involves accessing the abdominal cavity via one of the bodies' natural orifices, for example, mouth, anus, or vagina. This new surgical procedure is very appealing from patients' perspectives because it eliminates completely abdominal wall aggression and promises to reduce postoperative pain, in addition to all other advantages brought by laparoscopic surgery. However, the constraints imposed by both the mode of access and the limited technology currently available make NOTES very challenging for the surgeons. Redesign of the instruments is imperative in order to make this emerging operative access safe and reproducible. In this paper, we survey on the state-of-the-art devices used in NOTES and introduce both the flexible instruments based on improvement of current endoscopic platforms and the revolutionary concept of robotic platforms based on the convergence of communication and micromechatronics technologies. The advantages and limitations of each category are addressed. Potential solutions are proposed to improve the existing designs and develop robust and stable robotic platforms for NOTES.
\end{abstract}

\section{Introduction}

Past decades have evidenced a steady decrease in the invasiveness of surgical interventions. The first laparoscopic cholecystectomy was performed in the mid 1980s. The laparoscopic surgery, or minimally invasive surgery, was welcomed by the patients undergoing this alternative for the rapid recovery. The new procedures perform operations in the abdomen through small incisions, usually $0.5-1.5 \mathrm{~cm}$, which are much smaller than that needed by the traditional technique (over $10 \mathrm{~cm}$ ). Since the 1990s, the laparoscopic surgery gradually prevails over the traditional open surgery. It has been proved to decrease postoperative morbidity, shorten hospitalization and convalescence, and improve cosmesis while matching the outcomes of equivalent open procedures. Today, the majority of open surgical procedures have been replicated or even replaced by laparoscopic techniques.

At the same time, the endoscopy technique has been evolving from pure diagnostic devices to therapeutic devices thanks to the development of the microelectronic techniques.
In addition to the lighting and imaging parts, the endoscopes are designed to possess multiple working channels, enabling equipment and insertion of various instruments, such as the ultrasonic device, the laser cauter, the biopsy instruments, and polyp removal tools. It is not surprising that endoscopy and surgery would eventually work together, and this theoretic point of fusion is being turned into reality. With the objective of preventing port-site complications associated with laparoscopy, further decreasing discomfort and removing the scar on the body surface, Natural Orifice Transluminal Endoscopic Surgery (NOTES) has been proposed (Figure 1) [1].

NOTES involves accessing the abdominal cavity via one of the bodies' natural orifices, for example, mouth, anus, vagina, or urethra. A flexible endoscope is advanced into the peritoneal cavity after puncturing one of the viscera such as stomach, colon, vagina, or bladder. Conventional endoscopic instruments are introduced through the working channels of the endoscope in order to perform the operation. NOTES not only provides all the advantages of laparoscopic surgery but also offers several potential benefits, including 
cosmetic result, lower anesthesia requirements, less pain, even faster recovery, and a decreased incidence of woundrelated complications.

It is believed that NOTES may become the next major paradigm shift in surgery following laparoscopy [2]. Nevertheless, as an emerging surgical procedure, the safety issue of NOTES becomes a major concern. It requires continuous clinical practice and assessment and depends largely on the development of appropriate surgical tools. Since in literature there is a lack of a thorough survey on the instrumentation for NOTES, in this paper we hope to make up this gap by investigating the state-of-the-art instruments developed for NOTES, including both the prototype flexible endoscopes and the new concept robotic platforms. The paper is organized as follows: Section 2 introduces the background and status quo of NOTES; Section 3 and Section 4 present, respectively, the flexible endoscopes and the robotic platforms. The advantages and limitations are discussed, followed by potential solutions to the problems; Section 5 concludes the paper.

\section{Background of NOTES}

The first attempt of NOTES was reported in 2004 by Kalloo et al. in a porcine model [3]. They penetrated the gastric wall and operated in the abdominal cavity using an orally introduced flexible endoscope via a sterile overtube. The pioneer work demonstrated the feasibility and safety of an oral transgastric peritoneoscopy. The idea of "no-scar" abdominal surgery immediately captured the medical community and the general public. Since then, many researchers have used transluminal flexible endoscopy in animal models to perform various intraperitoneal procedures, ranging from tubal ligation to splenectomy [4].

In 2006, the first transgastric NOTES appendectomy performed in humans was reported by Rao and Reddy [5]. Swanström described the first case of human transgastric cholecystectomy in 2007 [6]. More and more clinical trials of NOTES commenced thereafter. To date, thousands of NOTES procedures have been carried out all around the world. Of all the natural orifices accessed by NOTES, the transvaginal route seems to be the most safe and feasible for clinical applications, because it minimizes the concerns about a secure enteral closure. The disadvantage is that it is possible only in the female. Sánchez-Margallo et al. reported transvaginal cholecystectomy performed in pig models without using laparoscopic assistance [7]. However, true NOTES, as an "incisionless" operation, has not been described in human clinical trials, because all cases reported were assisted by laparoscopic or percutaneous methods.

Before NOTES can be successfully and responsibly used in clinical care, several critical issues must be resolved, among which an appropriated instrumentation is of most importance [8-11]. Conventional flexible endoscopes are inadequate for performing complex transluminal surgical procedures. The limitations include the lack of a multitasking platform, the number and size of access channels, the inability to position and fix the instruments to allow robust

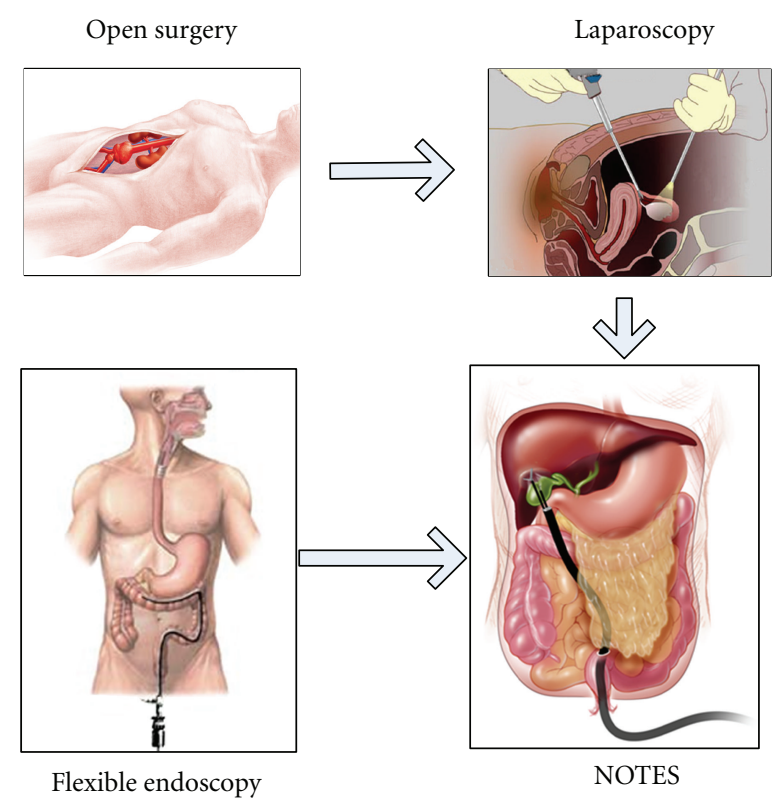

Figure 1: Natural orifice transluminal endoscopic surgery is a fusion of the therapeutic endoscopy and the laparoscopy surgery, which is a "scarless" surgery further decreasing the invasiveness.

retraction and exposure, and the unavailability of adequate triangulation, NOTES has encouraged a lot of research and development in the medical device industry. Many surgical devices are being developed, including advanced forms of endoscopes and robotic platforms. The details will be introduced in the following sections.

\section{Flexible Endoscopic Platforms}

3.1. Prototype of Endoscope. Flexible endoscopes are the main tool used in minimally invasive surgery. A typical endoscope is $10 \mathrm{~mm}$ in diameter and $70-180 \mathrm{~mm}$ in length. They are inserted directly into the hollow organ/cavity for examination or therapeutic treatment. Degani et al. [12] developed a $12 \mathrm{~mm}$ (diameter) $\times 300 \mathrm{~mm}$ (length) snake-like robot named "HARP". It consists of an inner and an outer snake made of rigid cylindrical links connected by spherical joint. By pulling/relaxing the cables stringing the joints, the probe can be made rigid/flexible, which is similar to the ShapeLock technology. Preliminary experiments in pigs with the prototype can reach the target, lock the outer snake, and replace the inner snake with colonoscope forceps. Abbott et al. [13] developed two generations of ViaCath systems for teleoperated endoluminal surgery. The endoluminal instruments consist of a connector, a flexible shaft, and an actuated joint with end-effector. The second generation instrument is $120 \mathrm{~cm}$ in length, $7.2 \mathrm{~mm}$ in diameter with a single lumen inside the shaft for the actuation cables, providing 9 degrees of freedom. The mechanical properties of the prototype have been evaluated. But it still needs validations with phantoms and animal models.

In comparison, the flexible endoscopic platforms for NOTES need to be stronger and more rigid to perform 
surgical procedures. Most prototypes are modified based on the therapeutic endoscopes by augmenting the number and the diameter of the channels. Swanström et al. partnered with USGI Medical developed Transport multilumen operating platform for NOTES [14, 15]. As shown in Figure 2, Transport is a $16 \mathrm{~mm}$ access device, with four large working channels, one for a standard $6 \mathrm{~mm}$ endoscope, and three others for large diameter instruments. It adopts the ShapeLock design, which allows independent steering of the tip and then lock into position once it is maneuvered to the operative site. The large $4 \mathrm{~mm}$ and $6 \mathrm{~mm}$ channels allow passage of stronger and flexible surgical tools. The force delivery at the tip can reach $0.89 \mathrm{~kg}$ and the instrument application force $0.1 \mathrm{~kg}[16]$.

Triangulation is one of the most essential concepts in laparoscopy, which means separation of the working hands from each other and to have the "eye" in-between the two "hands", as shown in Figure 3. USGI Medical developed a prototype called "Cobra" [15], which adds three independent arms controlled by a robotic interface to the Transport to provide some degree of triangulation, as shown in Figure 4.

Olympus modified a standard dual-channel therapeutic scope named the "R" scope for NOTES [17]. The multibending section of the scope allows it to be positioned near the target area and then locked. The device has two movable $3.8 \mathrm{~mm}$ instrument channels: one moves vertically and the other swings horizontally, which offer a reasonable simulation of triangulation, as shown in Figure 5. The two instruments can be manipulated during the operation with a knob and a lever that surround the angulation control knobs of the R-scope. Once the operator has decided on the knob or lever positions, these can be locked into position.

3.2. Limitations of Flexible Endoscope. The improved flexible endoscopes are useful for some antegrade intra-abdominal procedures. However, there still exist deficiencies due to their inherent features. One major point is that the flexibility makes it difficult to aggressively retract tissues. Due to the small channel size of the endoscopes, the end effectors of most instruments are small and feeble. A larger size and more ports permit some degree of triangulation but are still inadequate. Some instruments are fixed and require the device be removed to exchange tools and then reintroduced. In addition, the complexity of the devices does not allow a smooth and controlled movement of the tip and therefore the instrument, which challenges the precise maneuvers. The main limitations of the present flexible endoscope and the requirements for instruments used for NOTES are listed as follows [18-20].

(i) Platform stability: The inherent flexibility of current endoscopes impedes achieving a stable operation field for NOTES. The ideal instrument would be capable of atraumatic insertion and positioning but then be able to fix in position to free the surgeon's hands to manipulate multiple instruments.

(ii) Retraction: The lack of rigidity also limits the counter forces down the endoscope which can be applied to adequately retract tissues and apply strong sutures or clips. New methods need to be developed to allow vigorous traction and large organ retraction for exposure.

(iii) Triangulation: A critical concept in laparoscopy, which means separation of the working hands from each other and to have the "eye" in-between the two "hands". This is not the case for the flexible endoscope with inline instrumentation and optics. Independent movement of multichannel therapeutic instruments is desirable.

(iv) Size: The flexible endoscopes usually have a diameter of 5-15 mm with only one or two working channels. Each channel is $2-3 \mathrm{~mm}$ in diameter, which limits the size of surgical instruments and abilities to triangulate and maneuver tissue properly. The device for NOTES should contain at least 2 instrument channels in addition to the imaging part, to enlarge the range of motion and increase the degrees of freedom.

(v) Image: The image quality of current flexible endoscopes is comparable to laparoscopes for the most parts. However, there is an orientation problem when working in a retroflexed position, that is, the image might be inverted or reversed. An orthophoric imaging system with adequate lighting intensity is necessary to distinguish different anatomical structures in the intraperitoneal space.

Currently, the flexible endoscopes are not applicable for fine surgeries. There is a need to make significant improvements and design more aggressive instruments for NOTES. The problems mentioned above can be resolved to some extent with scope-handling expertise. However, a better long-term solution will be to redesign the endoscopic access devices and carry out the procedures in a completely new manner.

\section{Robotic Platforms}

At present, development of instrumentation to facilitate NOTES techniques is still in its infancy, but is critical for broadly applicable NOTES. Robotic technology is likely to allow us to make more agile and precise instruments than currently available tools, and perform procedures that cannot be done by conventional minimally invasive techniques. To address the limitations of the existing medical robots, such as bulky and expensive system, limited view field, and inconvenient operation, in recent years novel concepts of miniature/modular robots are being developed, which provide potential solutions to the laparoscopic and NOTES procedures. This section introduces the attempts on these robotic platforms.

4.1. Imaging Robot. Imaging robot is useful for providing visual feedback during the medical procedure. In the past decade, the wireless capsule endoscopy has been proved an established procedure for examination of the gastrointestinal tract [21]. The imaging robot encloses lighting, imaging, 


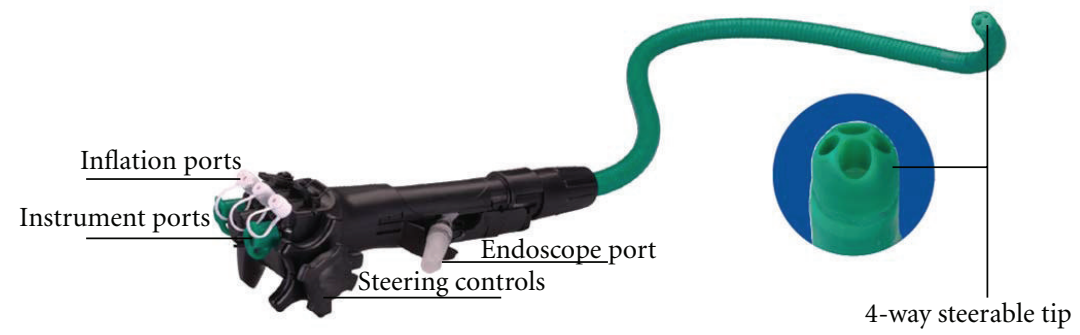

FIgURE 2: The shape-lock Transport endoscope with four operating channels (USGI Medical).

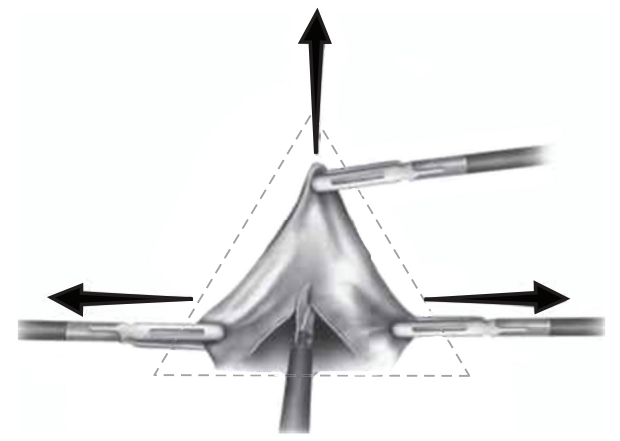

FIGURE 3: Demonstration of triangulation, a critical technique during laparoscopic surgery.

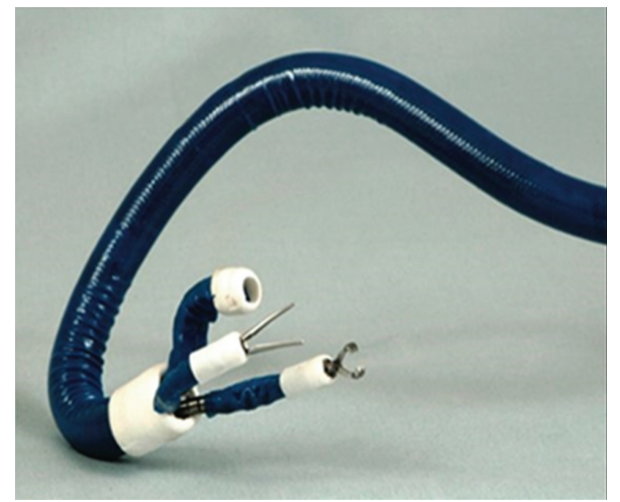

Figure 4: The Cobra triangulating scope (USGI Medical).

wireless transmission units, and button batteries within a capsule-sized of $11 \mathrm{~mm} \times 26 \mathrm{~mm}$. After swallowed, the capsule can take pictures and send them to the external data recorder wirelessly while travelling through the digestive tract by natural peristalsis. And at the completion of the examination, the capsule is excreted naturally. The wireless capsule endoscope enables completely noninvasive and painless examination. The swallowable feature and the technical breakthrough give inspirations for the design and development of medical microrobotics. Equipped with a guiding or an actuating mechanism, the capsule has great



Figure 5: The R-scope (Olympus Medical Systems).

potential to be applied for assisting the surgical operations $[22,23]$.

Rentschler et al. developed a mobile camera robot with a diameter of $12 \mathrm{~mm}$ and a length of $75 \mathrm{~mm} \mathrm{[24],} \mathrm{as} \mathrm{shown}$ in Figure 6. It consists of two wheels driven independently by $6 \mathrm{~mm} \mathrm{DC}$ motors to make forward, reverse, and turning motions. A tail is set in the middle of the wheels to prevent counter-rotation. An adjustable-focus image sensor is carried between the wheels to provide visual feedback during the movement of the robot. This robot has been tested in porcine model experiment, during which it was inserted into the peritoneal cavity through the transgastric incision, and retracted back through the esophagus by a standard upper endoscope after exploration of the abdominal cavity.

Rentschler and Oleynikov also introduced a fixed-base imaging robot with a body of $15 \mathrm{~mm}$ diameter. It is mounted on a spring-loaded foldable-tripod platform that allows a 45-degree angle forward tilting and 360-degree panning [25]. The objective is to enhance visualization and provide indepth perception of the abdominal cavity. The three legs can be retracted during insertion and abducted by the torsion springs after entry. Light-emitting diodes (LEDs) are equipped to provide illumination. The robot has been evaluated in canine and porcine model experiments to provide augmented visual feedback and enhance the field 


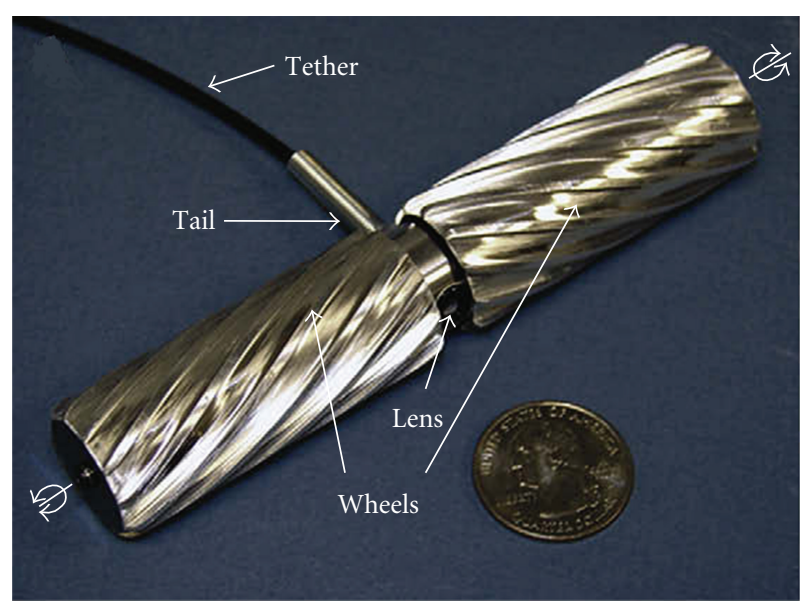

Figure 6: Picture of a mobile imaging robot.

of view. To avoid the tether and facilitate the operation, a wireless prototype is under development.

Magnetic anchoring enables position and reposition of the instruments without requirement of invasive access ports. This technique is also useful for reducing the instrument conflict during the surgical procedures. The particular advantages are attracting more and more attention for the development of the surgical robotics. Lehman et al. developed a repositionable platform for visualization, which can be attached to the interior abdominal wall by magnetic attraction [26]. As shown in Figure 7, the cylindrical peritoneum-mounted imaging robot consists of a stationary outer tube and a rotating inner tube housing the imaging unit and three DC micromotors. Two permanent magnets are embedded at each end of the robot, which can interact with a magnetic handle located on the exterior abdominal wall. Actuated by the micromotors, the camera can make panning and tilting motions and provide nonstereo visual feedback to the surgical system. This robot was tested in a nonsurvival cholecystectomy in a porcine model, working cooperatively with the da Vinci Surgical System (Intuitive Surgical company). In further optimized design under the same framework, the robot has a more compact size with a diameter of $12 \mathrm{~mm}$ and contains LEDs for lighting. One single micromotor is enclosed to tilt the camera, and the external magnetic handle takes charge of both positioning and panning of the camera.

Cadeddu et al. packaged a commercially available miniature imager and LEDs with biocompatible enclosure to fabricate a magnetically anchored camera [27]. An NdFeB magnet is enveloped in the $20 \mathrm{~mm} \times 60 \mathrm{~mm}$ profile to generate coupling force, which can lift up to $45 \mathrm{~g}$ at a distance of $30 \mathrm{~mm}$. By manipulation of the external guiding unit, the camera can be guided to slide and make yaw and pitch movement. Once the system reaches the optimal location, it can be fixed on the abdominal wall with a needle. Initial clinical experience showed the technical feasibility and the effectiveness of the camera system.

The robots mentioned above can assist the surgical procedure by providing additional visualization; however, their

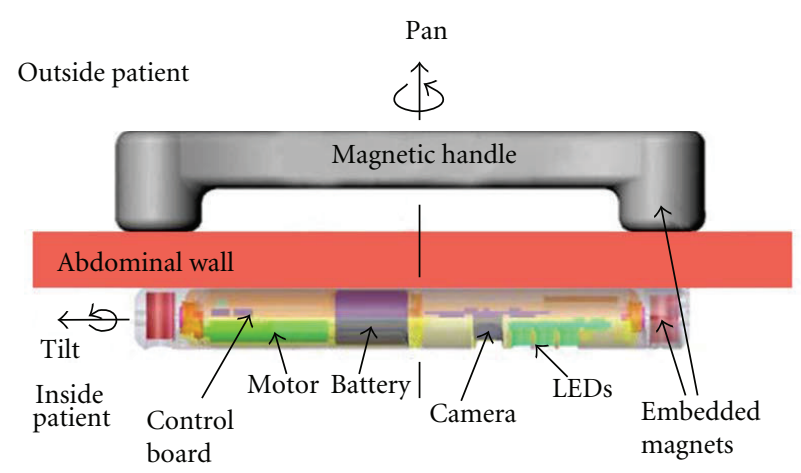

FIGURE 7: Schematic of a repositionable visualization platform.

functions are limited without equipment of other operative instruments. Multifunctional platform is desirable to offer task assistance such as tissue manipulation, or even perform complete NOTES procedures. The microrobots developed for this purpose will be introduced in the following sections.

4.2. Operative and Cooperative Robot. Besides the imaging module, operation tools are being equipped to the miniature robot to make it more versatile. Shah et al. introduced a multiarmed robot with stereovision to provide a repositionable platform for performing NOTES procedures inside the peritoneal cavity [28]. As depicted in Figure 8, the robot consists of two arms (53 mm length, $14 \mathrm{~mm} \times 17 \mathrm{~mm}$ crosssection) connected to a body ( $80 \mathrm{~mm}$ length) by rotational shoulder joints. Each arm is extensile for $0-32 \mathrm{~mm}$ and fitted with an end-effector, for example, a grasper and a cautery. The body contains two cameras to provide stereo vision and a permanent magnet to hold the robot to the upper abdominal wall by an external magnetic handle. It can also be repositioned by the external magnetic handle to get suitable view field without additional incision. Nonsurvival porcine model experiments showed the robot had the ability to retract the tissue by the grasper and perform small bowel dissection by the cautery $[29,30]$.

In minimally invasive surgery such as NOTES, the insertion of the operation tools is severely limited by the size of the natural orifices. Therefore, the tools need to be miniaturized to negotiate through the geometry of the natural lumen. Instead of integrating all the tools into one microrobot, one way to solve the problem is to use multiple microrobots to carry out cooperative operation, that is, to develop cooperative microrobots to meet the requirement. Each microrobot takes charge of only one specific task, by which the structure of the robot can be made compact and simple. By fixing multiple forceps and retractors to small internal magnets and maneuvering them by large extracorporeal magnet, it is possible to achieve noninvasive retraction and triangulation inside the abdominal cavity, similar to the traditional laparoscopy. Besides, the easy repositioning of the devices makes the whole system reconfigurable. The magnetic manipulation of two forceps for triangulation has been successfully performed in single-port laparoscopic cholecystectomy by Dominguez et al. [31] 


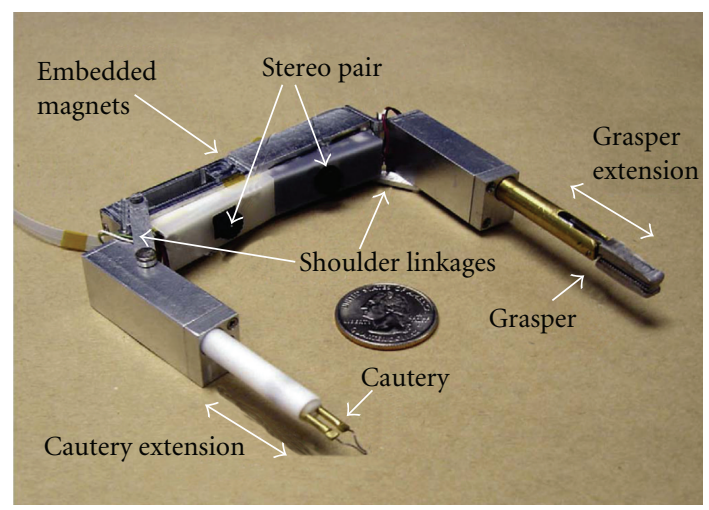

Figure 8: Picture of an operative robot with two arms.

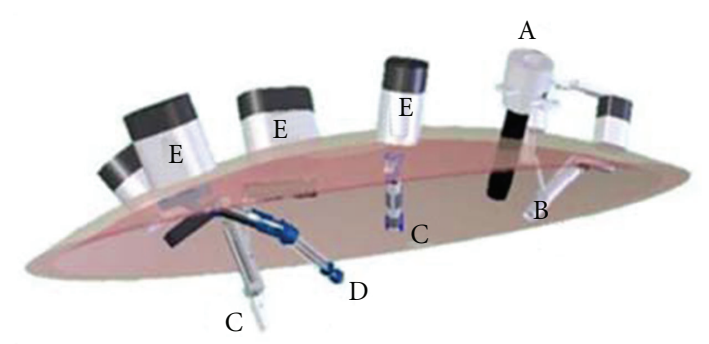

FIGURE 9: Schematic representation of MAGS platform. (A) conventional deployment trocar; (B) MAGS camera; (C) retractors; (D) robotic cauterizer; and (E) external magnets.

Shah et al. reported a nonsurvival NOTES procedure in a porcine model using a standard upper gastrointestinal endoscope with three cooperative microrobots, including the peritoneum-mounted imaging robot introduced in Section 4.1, a lighting robot carrying six white LEDs, and a retraction robot consisting of a grasper tethered with a DC motor [28]. All the microrobots were secured along the upper abdominal wall by external magnetic handles. This procedure demonstrated the feasibility of providing a stable repositionable platform for NOTES using multiple miniature in vivo robots.

Park et al. developed a "transabdominal magnetic anchoring and guidance system" (MAGS), which can be used to control an intra-abdominal laparoscope and multiple working instruments introduced through a single $15 \mathrm{~mm}$ port [32]. As shown in Figure 9, the MAGS incorporates an internal camera system, two types of passive tissue retractors and a pneumatically controlled robotic arm attached with a hook cauterizer. The $158 \mathrm{~mm}$ long robotic arm has three degrees of freedom, with the first joint and the second joint capable of 50 degree and 45 degree of motion, respectively, and the third joint allowing $20 \mathrm{~mm}$ additional extension of the arm. The camera and robotic arm are tethered to their external controller. All the MAGS instruments are affixed to the abdominal wall using multiple external magnetic anchors during the surgery. Zeltser et al. reported positive results in single trocar laparoscopic nephrectomy using MAGS in two nonsurvival porcine models [33]. Scott et al. conducted

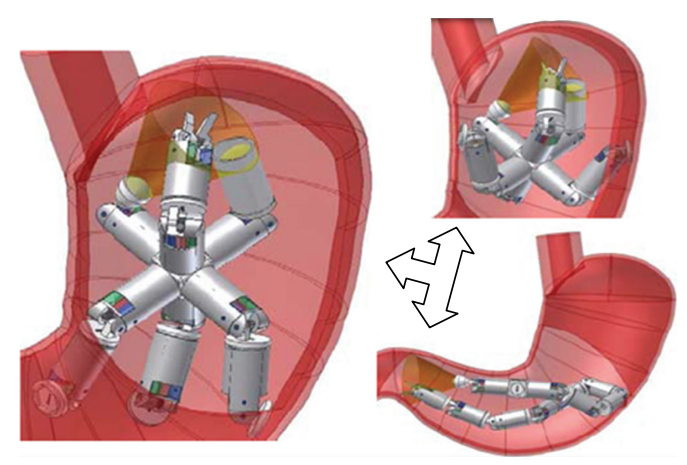

FIGURE 10: Conceptual representation of configurable modular robots inside the stomach.

transvaginal NOTES cholecystectomy in four nonsurvival porcine models and completed the procedures in two of them using only NOTES/MAGS approach [34]. Best et al. have experimented the MAGS on pigs with mean $2.1 \mathrm{~cm}$ thick abdominal wall sections. The histologic assessment showed that MAGS do not appear to cause tissue damage when coupled across porcine abdominal walls for up to 4 hours [35]. The findings support the further development of magnetic instruments to be used in humans, which facilitate the NOTES procedure while alleviating shortcomings of the flexible platforms.

4.3. Self-Assembled Robot. The self-organized modular robots were proposed by Fukuda et al. in 1989 [36]. For a long time this kind of robot was developed for exploration and surveillance, for which the working space usually placed no restriction on the size and number of the modules. This concept has not been applied to the surgical use until recent years when wireless reconfigurable robots were proposed for endoluminal surgery [37-39]. Figure 10 shows the conceptual design. Each modular robot has an ingestible size and can be ingested/inserted into the lumen through the natural orifices/endoscope channels. After reaching the working space, the robots start the self-assembling process by magnetic force. An external controller is operated by the surgeon to make wireless control of the robotic configuration, the assembly, and the surgical task. Usually a group of modular robots consists of a central module, multiple structure modules, and functional modules. The structure modules are used to link the functional modules for diagnosis and/or intervention with the central module. The same group of modular robots can be assembled into several different topologies inside the abdominal cavity. After completion of the surgical procedure, the robots either resemble into a snake shape or disassemble into individual modules and excrete naturally. The self-assembly design not only reduces the size of each module, but also enables convenient adding and replacement of different interventional functions/modules even during the operation.

Harada et al. prototyped the structure modules and the biopsy module $[38,39]$. In spite of the different distal designs, the two modules share the same components of a brushless DC motor, a control board, an assembling magnet, 
and a battery. The structure module has a motor actuated joint, which can make \pm 90 degree bending with a torque of $6.5 \mathrm{mN} \cdot \mathrm{m}$ and 0 degree to 180 degree rotation with a torque of $2.2 \mathrm{mN} \cdot \mathrm{m}$. The biopsy module has a foldable grasping mechanism, driven by the motor, to miniaturize the size during insertion and generate the grasping force for tissue sampling.

The modular robots are easy to be assembled into different topologies by magnetic attraction, while the disassembly method is a much more complex problem. Diller et al. [40] proposed to control the assembly and disassembly using an electrostatic anchoring surface, which can selectively keep specific modules from moving. The work is inspiring although the dimensions of the modules in the proposed work are in $1 \mathrm{~mm}$ and the system works in 2D surface, which is not appropriate for surgical applications.

4.4. Limitations of Robotic Platform. Comparing with the flexible endoscopes, the robotic approach facilitates the operations for minimally invasive surgery such as NOTES by allowing the use of multiple surgical instruments, improving triangulation and ergonomics, and providing relatively stable and rigid platform. The modular and cooperative design simplifies the structure of each individual functional robot and miniaturizes the size to enable the passage through a single port. Experimental evaluations confirm the effectiveness of the microrobots in providing extra visualization and task assistance $[24,26]$. Nevertheless, the in vivo microrobots are all in nonsurvival animal evaluation stage and not mature enough for clinical use. While this approach is promising, the technology needs to address current limitations and be further developed to ease the operation and ensure safety in the future. Some limitations and potential solution are listed as follows.

(i) Platform stability: Most imaging and operative microrobots are anchored on the abdominal wall by magnetic coupling to avoid extra incisions. However, the magnetic attraction force diminishes exponentially with respect to the distance between the internal magnetic joint and the external handle. Experimental studies show that the force will be inadequate to retract the tissue at a distance larger than $15 \mathrm{~mm}$, resulting in unstable platform in humans with thicker abdominal walls. An alternative way is to exchange the magnetic anchor by needles after the microrobots are guided and deployed magnetically inside the abdominal cavity. Obviously this will lead to a longer learning curve and less dexterity of the system. Stronger magnetic field is preferable to provide secure anchoring of the microrobot. For the reconfigurable microrobot, except the robust assembly by the magnetic joints, the undocking method is also critical for breaking up the topology and releasing the modules. Properly designed electromagnetic field may be feasible to generate powerful while controllable attraction force. Other than the magnetic anchoring problem, there are also reports about failure of the mechanical parts during experiments. Besides using light but strong materials to handle this problem, parallel mechanical design may carry more force than the serial one.

(ii) Wireless control: The imaging and motorized microrobots introduced in literature are all tethered to the external power and control system. Future platform may be optimized by incorporating an on-board power supply and wireless controller, which enables completely independent deployment of each individual microrobot and reduces the possible confliction among different microrobots and other instruments. The technology of wireless capsule endoscope offers successful experiences that can be adopted for nextgeneration self-contained microrobot.

(iii) Versatile and robust operation tools: The operative microrobots reported mainly consist of some basic operation tools such as the grasper and the cautery, so that most microrobots can only work with the flexible endoscope as an assistant. Equipment of more end effectors such as scissors, needle driver, and dissector will broaden the range of the operations. Besides, robust graspers with increased rigidity, bigger jaws, and better control over positioning are desired especially for the clinical settings of thickened or diseased tissues. The image quality of the camera robot needs to be improved to match that of the conventional laparoscopes and flexible endoscopes. More integration of robotic control systems will facilitate additional functionality of the surgical system. Ultimately, it is expected that completely independent operations be performed by the versatile microrobots.

(iv) Articulated instruments: In the operative microrobots, tools are attached directly to the magnetic joint which is coupled to the external magnetic handle. The advantage of the design lies in simple structure and compact size. However, one degree of freedom confines the operation in a small working space and thus restricts performance of some complicated operations. Multiple micro DC motors may be equipped to actuate the articulated instrument, providing extended workspace and better dexterity.

In summary, each system has its advantages and limitations. It is not obvious which system is uniquely superior to the others in terms of the evaluation based on size, image quality, maneuverability, stability, and ability to provide triangulation. Doctors and engineers are still exerting their efforts on improvement and novel design of the robotic systems.

\section{Conclusions}

Like the introduction of laparoscopic procedures, which have great impact on the surgical treatment in the past 30 years, NOTES may become another paradigm shift of the surgery. However, before the wide adoption of the novel procedure, clinical and engineering limitations must 
be addressed. One critical demand is the development of new operation platforms. At present, most clinical trials have been performed with conventional laparoscopic instruments or flexible endoscopes revised based on the gastrointestinal endoscopes. Nevertheless, the former can only be employed in specific transvaginal operations and the latter platforms lack rigidity and cannot fulfill the requirements of triangulation and retraction. New-concept instruments need to be developed for NOTES, among which robotics provide a promising way. Pilot study has validated the feasibility of using operative and imaging microrobots for task assistance. Additional investigations are to be carried out to evaluate the outcomes in survival models in animals and ultimately safety and efficacy in humans. With more advanced robotics proposed and developed, we can expect that NOTES procedures become more mature and more widely accepted in the future.

\section{References}

[1] S. S. Garud and F. F. Willingham, "Natural orifice transluminal endoscopic surgery," Endoscopy, vol. 44, no. 9, pp. 865-868, 2012.

[2] S. V. Kantsevoy, B. Hu, S. B. Jagannath et al., "Transgastric endoscopic splenectomy: is it possible?" Surgical Endoscopy, vol. 20, no. 3, pp. 522-525, 2006.

[3] A. N. Kalloo, V. K. Singh, S. B. Jagannath et al., "Flexible transgastric peritoneoscopy: a novel approach to diagnostic and therapeutic interventions in the peritoneal cavity," Gastrointestinal Endoscopy, vol. 60, no. 1, pp. 114-117, 2004.

[4] L. L. Swanstrom, Y. Khajanchee, and M. A. Abbas, "Natural orifice transluminal endoscopic surgery: the future of gastrointestinal surgery," The Permanente Journal, vol. 12, no. 2, pp. 42-47, 2008.

[5] G. V. Rao and N. Reddy, "Transgastric appendectomy in humans," in Proceedings of the Society of American Gastrointestinal and Endoscopic Surgeons (SAGES) Annual Conference, Dallas, Tex, USA, 2006.

[6] L. L. Swanström, "Natural orifice transluminal endoscopic surgery," Endoscopy, vol. 41, no. 1, pp. 82-85, 2009.

[7] F. M. Sánchez-Margallo, J. M. Asencio, M. C. Tejonero et al., "Technical feasibility of totally natural orifice cholecystectomy in a swine model," Minimally Invasive Therapy and Allied Technologies, vol. 17, no. 6, pp. 361-364, 2008.

[8] J. Pearl and J. Ponsky, "Natural orifice transluminal endoscopic surgery: past, present and future," Journal of Minimal Access Surgery, vol. 3, no. 2, pp. 43-46, 2007.

[9] L. L. Swanstrom, M. Whiteford, and Y. Khajanchee, "Developing essential tools to enable transgastric surgery (NOTES)," Surgical Endoscopy, vol. 22, no. 3, pp. 16-20, 2008.

[10] S. J. Bardaro and L. Swanström, "Development of advanced endoscopes for natural orifice transluminal endoscopic surgery (NOTES)," Minimally Invasive Therapy and Allied Technologies, vol. 15, no. 6, pp. 378-383, 2006.

[11] D. Rattner and A. Kalloo, "White paper-ASGE/SAGES working group on natural orifice translumenal endoscopic surgery," Surgical Endoscopy, vol. 20, no. 2, pp. 329-333, 2006.

[12] A. Degani, H. Choset, A. Wolf, and M. A. Zenati, "Highly articulated robotic probe for minimally invasive surgery," in Proceedings of the IEEE International Conference on Robotics and Automation (ICRA '06), pp. 4167-4172, Orlando, Fla, USA, May 2006.
[13] D. J. Abbott, C. Becke, R. I. Rothstein, and W. J. Peine, "Design of an endoluminal NOTES robotic system," in Proceedings of the IEEE/RSJ International Conference on Intelligent Robots and Systems (IROS '07), pp. 410-416, San Diego, Calif, USA, October 2007.

[14] J. P. Pearl and J. L. Ponsky, "Natural orifice translumenal endoscopic surgery: a critical review," Journal of Gastrointestinal Surgery, vol. 12, no. 7, pp. 1293-1300, 2008.

[15] http://www.usgimedical.com/eos/index.htm .

[16] L. Swanström, P. Swain, and P. Denk, "Development and validation of a new generation of flexible endoscope for NOTES," Surgical Innovation, vol. 16, no. 2, pp. 104-110, 2009.

[17] J. Yonezawa, M. Kaise, K. Sumiyama, K. Goda, H. Arakawa, and H. Tajiri, "A novel double-channel therapeutic endoscope ("R-scope") facilitates endoscopic submucosal dissection of superficial gastric neoplasms," Endoscopy, vol. 38, no. 10, pp. 1011-1015, 2006.

[18] L. L. Swanstrom, R. Kozarek, P. J. Pasricha et al., "Development of a new access device for transgastric surgery," Journal of Gastrointestinal Surgery, vol. 9, no. 8, pp. 1129-1137, 2005.

[19] R. A. Cahill, "Natural orifice transluminal endoscopic surgery-here and now," The Surgeon, vol. 8, no. 1, pp. 44-50, 2010.

[20] M. C. Meadows and R. S. Chamberlain, "A review on the status of natural orifice transluminal endoscopic surgery (NOTES) cholecystectomy: techniques and challenges," Open Access Surgery, vol. 3, pp. 73-86, 2010.

[21] G. Iddan, G. Meron, A. Glukhovsky, and P. Swain, "Wireless capsule endoscopy," Nature, vol. 405, no. 6785, pp. 417-418, 2000.

[22] X. Wang and M. Q. H. Meng, "A magnetic stereo-actuation mechanism for active capsule endoscope," in Proceedings of the 29th Annual International Conference of IEEE-EMBS, Engineering in Medicine and Biology Society (EMBC '07), pp. 2811-2814, Lyon, France, August 2007.

[23] M. Quirini, A. Menciassi, S. Scapellato et al., "Feasibility proof of a legged locomotion capsule for the GI tract," Gastrointestinal Endoscopy, vol. 67, no. 7, pp. 1153-1158, 2008.

[24] M. E. Rentschler, J. Dumpert, S. R. Platt, S. M. Farritor, and D. Oleynikov, "Natural orifice surgery with an endoluminal mobile robot," Surgical Endoscopy, vol. 21, no. 7, pp. 12121215, 2007.

[25] M. E. Rentschler and D. Oleynikov, "Recent in vivo surgical robot and mechanism developments," Surgical Endoscopy, vol. 21, no. 9, pp. 1477-1481, 2007.

[26] A. C. Lehman, K. A. Berg, J. Dumpert et al., "Surgery with cooperative robots," Computer Aided Surgery, vol. 13, no. 2, pp. 95-105, 2008.

[27] J. Cadeddu, R. Fernandez, M. Desai et al., "Novel magnetically guided intra-abdominal camera to facilitate laparoendoscopic single-site surgery: initial human experience," Surgical Endoscopy, vol. 23, no. 8, pp. 1894-1899, 2009.

[28] B. C. Shah, S. L. Buettner, A. C. Lehman, S. M. Farritor, and D. Oleynikov, "Miniature in vivo robotics and novel robotic surgical platforms," Urologic Clinics of North America, vol. 36, no. 2, pp. 251-263, 2009.

[29] A. C. Lehman, J. Dumpert, N. A. Wood et al., "Natural orifice cholecystectomy using a miniature robot," Surgical Endoscopy, vol. 23, no. 2, pp. 260-266, 2009.

[30] S. R. Platt, J. A. Hawks, and M. E. Rentschler, "Vision and task assistance using modular wireless in vivo surgical robots," IEEE Transactions on Biomedical Engineering, vol. 56, no. 6, pp. 1700-1710, 2009. 
[31] G. Dominguez, L. Durand, J. de Rosa, E. Danguise, C. Arozamena, and P. A. Ferraina, "Retraction and triangulation with neodymium magnetic forceps for single-port laparoscopic cholecystectomy," Surgical Endoscopy, vol. 23, no. 7, pp. 16601666, 2009.

[32] S. Park, R. A. Bergs, R. Eberhart, L. Baker, R. Fernandez, and J. A. Cadeddu, "Trocar-less instrumentation for laparoscopy: magnetic positioning of intra-abdominal camera and retractor," Annals of Surgery, vol. 245, no. 3, pp. 379-384, 2007.

[33] I. S. Zeltser, R. Bergs, R. Fernandez, L. Baker, R. Eberhart, and J. A. Cadeddu, "Single trocar laparoscopic nephrectomy using magnetic anchoring and guidance system in the porcine model," The Journal of Urology, vol. 178, no. 1, pp. 288-291, 2007.

[34] D. J. Scott, S. J. Tang, R. Fernandez et al., "Completely transvaginal NOTES cholecystectomy using magnetically anchored instruments," Surgical Endoscopy, vol. 21, no. 12, pp. 2308-2316, 2007.

[35] S. L. Best, W. Kabbani, D. J. Scott et al., "Magnetic anchoring and Guidance system instrumentation for laparo-endoscopic single-site surgery/natural orifice transluminal endoscopic surgery: lack of histologic damage after prolonged magnetic coupling across the abdominal wall," Urology, vol. 77, no. 1, pp. 243-247, 2011.

[36] T. Fukuda, S. Nakagawa, Y. Kawauchi, and M. Buss, "Structure decision method for self organising robots based on cell structures-CEBOT," in Proceedings of the IEEE International Conference on Robotics and Automation, pp. 695-700, Scottsdale, Ariz , USA, May 1989.

[37] E. Susilo, P. Valdastri, A. Menciassi, and P. Dario, "A miniaturized wireless control platform for robotic capsular endoscopy using advanced pseudokernel approach," Sensors and Actuators A, vol. 156, no. 1, pp. 49-58, 2009.

[38] K. Harada, E. Susilo, A. Menciassi, and P. Dario, "Wireless reconfigurable modules for robotic endoluminal surgery," in Proceedings of the IEEE International Conference on Robotics and Automation, pp. 2699-2704, Kobe, Japan, May 2009.

[39] K. Harada, S. Russo, T. Ranzani, A. Menciassi, and P. Dario, "Design of Scout Robot as a robotic module for symbiotic multi-robot organisms," in Proceedings of the International Symposium on Micro-NanoMechatronics and Human Science, November 2011.

[40] E. Diller, C. Pawashe, S. Floyd, and M. Sitti, "Assembly and disassembly of magnetic mobile micro-robots towards deterministic 2-D reconfigurable micro-systems," The International Journal of Robotics Research, vol. 30, no. 14, pp. 1667-1680, 2011. 

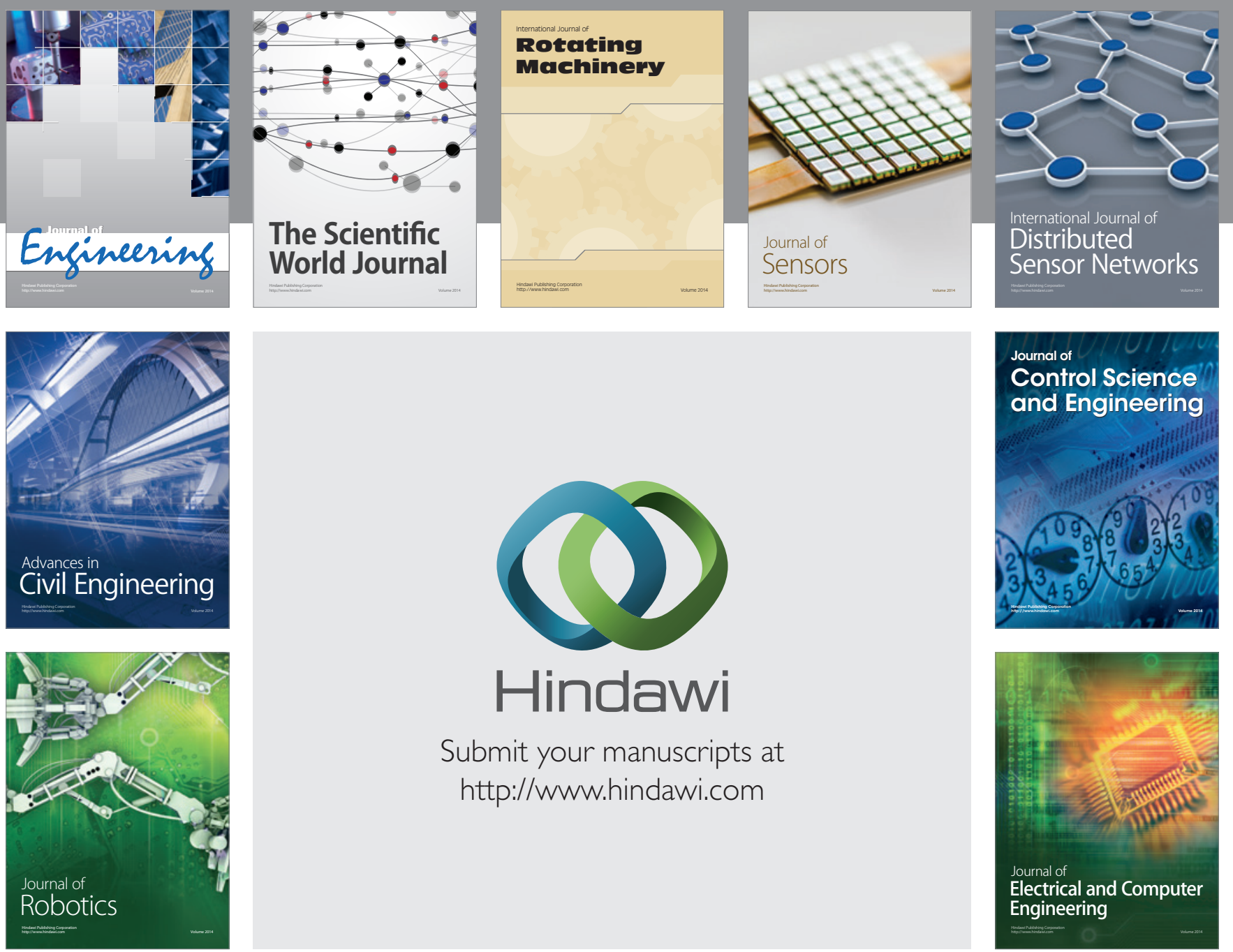

Submit your manuscripts at

http://www.hindawi.com
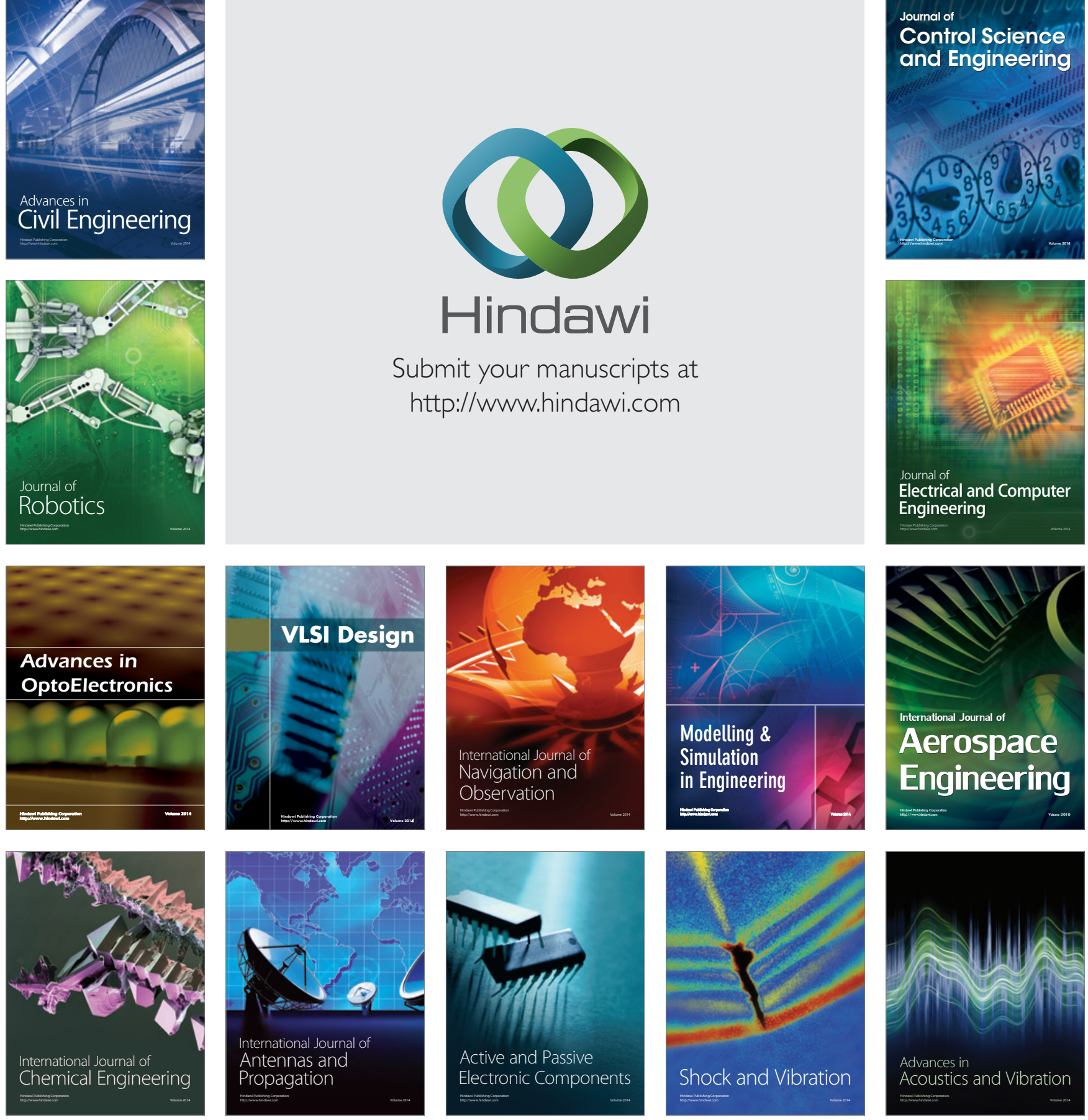